\title{
Formulation of non-ionic surfactant vesicles (NISV) prepared by microfluidics for therapeutic delivery of siRNA into cancer cells
}

\author{
Mohammad A. Obeid ${ }^{1,2 *}$, Ashref Elburi' ${ }^{1}$, Louise C. Young' ${ }^{1}$ Alexander B. Mullen${ }^{1}$, \\ Rothwelle J. Tate ${ }^{1}$, Valerie A. Ferro ${ }^{1 *}$ \\ ${ }^{1}$ Strathclyde Institute of Pharmacy and Biomedical Sciences, University of Strathclyde, 161 \\ Cathedral Street, G4 0RE Glasgow, United Kingdom. \\ ${ }^{2}$ Faculty of Pharmacy, Yarmouk University, Irbid, Jordan. \\ *Corresponding authors. E-mails Addresses: mohmad-ali-radi-obid@strath.ac.uk, \\ v.a.ferro@strath.ac.uk. Tel: +447512373361,+44 (0)1415483724.
}

\begin{abstract}
Abbreviations
NISV: Non-ionic surfactant vesicles; CN: cationic NISV; PBS: Phosphate buffered saline; MPG: Monopalmitin glycerol; Chol: Cholesterol; T85: Tween 85; PDI: Polydispersity index;

ZP: Zeta potential; siRNA: Small interfering RNA; RNAi: RNA interference; GFP: Green Fluorescent Protein; FACS: fluorescence-activated cell sorter.
\end{abstract}




\section{Table of contents graphic}
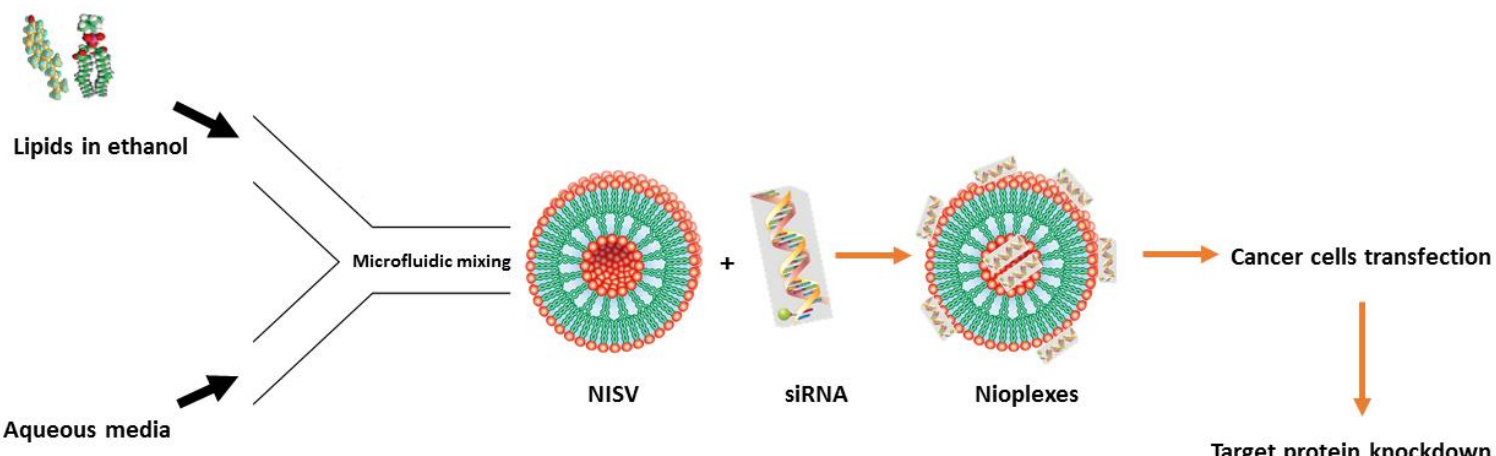

Target protein knockdown 


\begin{abstract}
Small interfering RNAs (siRNA) have a broad potential as therapeutic agents to reversibly silence any target gene of interest. The clinical application of siRNA requires the use of safe and effective delivery systems. In this study, we investigated the use of non-ionic surfactant vesicles (NISV) for the delivery of siRNA. Different types of NISV formulations were synthesised by microfluidic mixing and then evaluated for their physiochemical properties and cytotoxicity. The ability of the NISV to carry and transfect siRNA targeting green fluorescent protein (GFP) into A549 that stably express GFP (copGFP-A549) was evaluated. Flow cytometry and Western blotting were used to study the GFP expression knockdown and significant knockdown was observed as a result of siRNA delivery to the cells by NISV. This occurred in particular when using tween 85 , which was able to achieve more than $70 \%$ GFP knockdown. NISV were thus demonstrated to provide a promising and effective platform for therapeutic delivery of siRNA.
\end{abstract}

\title{
Key Words
}

Non-ionic surfactant vesicles, Microfluidics, RNA interference, Drug delivery 


\section{Introduction}

The discovery of the RNA interference (RNAi) mechanism by Fire et al. represents one of the most significant advances in molecular biology. This mechanism is an endogenous posttranscriptional gene regulatory process that involves the degradation of messenger RNA (mRNA) in a highly sequence-specific manner [1]. Double-stranded (ds) small interfering RNA (siRNA), composed of 21-23 base pairs in length, can induce RNAi and interfere with the expression of the protein of the target RNA [2]. The RNAi mechanism involves the incorporation of the anti-sense strand of the siRNA into the RNA-induced silencing complex in the cytoplasm, followed by cleavage of the target mRNA that is complementary to the antisense strand of the siRNA to cause silencing of the gene encoded by that mRNA [3]. Following this discovery, a significant body of research has been carried out to investigate the application of RNAi for the treatment of human diseases [4, 5]. For therapeutic applications, synthetic siRNA can be designed to target overexpressed genes in human diseases such as cancer [6]. The potential application of siRNA therapeutics has been demonstrated in different types of cancer, including bladder, ovarian, lung, and prostate cancers [7-10]. However, the successful application of siRNA-based therapeutics is dependent on the efficient delivery of siRNA to target cells [11]. Naked siRNA has a very short half-life as a result of rapid degradation and elimination from the blood by nucleases [12]. Moreover, the negative charge of siRNA molecules prevents their passive diffusion across hydrophobic cellular membranes in addition to creating electrostatic repulsion with negatively charged cellular membrane proteins [13]. Due to the poor stability of siRNA in physiological fluids and inefficient cellular uptake, effective delivery of therapeutic siRNA into the cytoplasm of target cells is one of the main challenges in the development of siRNAbased treatments [14]. An ideal siRNA delivery system, for clinical application, should protect the siRNA from rapid digestion, efficiently deliver the therapeutic siRNA into the 
target cells, and promote the subsequent release of siRNA from endosome vesicles into the cytoplasm, where they can be loaded into the silencing complex for gene silencing. Moreover, the delivery system should be biodegradable with low toxicity.

Non-viral delivery systems such as liposomes, dendrimers, cell-penetration peptides, and many others have been investigated for the delivery of siRNA to expand their clinical application [15]. Lipid-based nanoparticles such as liposomes are by far the most studied drug-delivery system for this purpose. However, due to the limitations of liposomes, such as cost and stability, the delivery of siRNA by non-ionic surfactant vesicles (NISV) have been investigated as an alternative. NISV are lipid-based nanoparticles, which are composed of non-ionic surfactants, cholesterol, and charged lipids. These components self-assemble upon hydration into a bilayer structure enclosing an aqueous core similar to liposomes [16]. NISV are generally superior to liposomes in terms of stability and production costs [17]. Different types of surfactant can be used in NISV formulations such as Tweens, Spans, Brijs, and many others [18]. NISV have been widely investigated as drug delivery systems due to their potential to carry and encapsulate a variety of therapeutic agents. However, the application of NISV in the field of gene delivery has not been investigated extensively. In terms of nucleic acid delivery, most of the research in the literature use these vesicles for DNA delivery [19, 20], and there is only a limited number of publications describing their use for siRNA delivery [21-23].

Previously, stable NISV through microfluidics were formulated [18]. In this study, we have synthesised cationic NISV (CN) using microfluidics with different surfactants and investigated their potential application for siRNA transfection. The cationic charge on the formulated NISV was achieved using the cationic lipid didodecyldimethylammonium bromide (DDAB), which not only helps with constructing the NISV bilayer structure, but also offers positive charge to both bind siRNA drugs and mediate cellular uptake via 
electrostatic adhesion to cellular surfaces that carry a slight negative charge. The prepared $\mathrm{CN}$ were evaluated for their physical characteristics, cytotoxicity, and transfection efficiency. Results were compared against a commercially available transfecting reagent, HiPerFect (Qiagen, UK).

\section{Materials and Methods}

\subsection{Materials}

Monopalmitin glycerol (Monohexadecanoin) (MPG) was purchased from Larodan Fine Chemicals AB (Sweden). Cholesterol (Chol); polyoxyethylenesorbitan trioleate (Tween 85); DDAB; resazurin powder; Tris base; sodium pyrophosphate $\left(\mathrm{Na}_{4} \mathrm{P}_{2} \mathrm{O}_{7}\right)$; sodium dodecyl sulphate (SDS); bromophenol blue; glycerol; glycine; sodium chloride $(\mathrm{NaCl})$; bovine serum albumin (BSA); ethylenediamine tetra-acetic acid (EDTA); Tween 20; Dulbecco's Modified Eagles medium (DMEM); L-glutamine; and penicillin-streptomycin were purchased from Sigma-Aldrich (UK). Foetal bovine serum (FBS) was purchased from Biosera (UK). Sodium pyruvate $(100 \mathrm{mM})$ and minimum essential medium non-essential amino acids (MEM NEAA) were purchased from Life Technologies (UK). Skimmed milk powder was purchased from Premier Foods Ltd (UK). The human non-small cell lung cancer (A549) cell line was purchased from the American Type Culture Collection (ATCC®). Human non-small cell lung cancer A549 cells that stably express Green Fluorescent Protein (copGFP-A549) were purchased from Cell Biolabs, Inc., (UK). Sterile, RNase-free phosphate buffered saline 1M and sterile RNase-free water were purchased from LONZA (UK). AllStars AF488-labelled Negative Control siRNA and HiPerFect transfecting reagent were purchased from Qiagen (UK). The anti-GFP DsiRNA (siGFP) duplex sequence (1), and the non-targeting scrambled DsiRNA (2) (Table 1) were synthesised by Integrated DNA Technologies (Belgium). A rabbit polyclonal antibody against copGFP was purchased from Evrogen JSC (Russia) and a 
rabbit polyclonal antibody against glyceraldehyde 3-phosphate dehydrogenase (GAPDH) was purchased from Santa Cruz Biotechnology (UK).

Table 1. Sequences of siGFP (1) and non-targeting scrambled DsiRNA (2).

\begin{tabular}{|l|c|r|}
\hline \multicolumn{2}{|c|}{ siRNA sequence (5'-3') } \\
\hline \multirow{2}{*}{$\mathbf{1}$} & Sense & rCrGrCrArUrGrArCrCrArArCrArArGrArUrGrArArGrArGCA \\
\cline { 2 - 4 } & Antisense & rUrGrCrUrCrUrUrCrArUrCrUrUrGrUrUrGrGrUrCrArUrGrCrGrGrC \\
\hline \multirow{2}{*}{$\mathbf{2}$} & Sense & rCrGrUrUrArArUrCrGrCrGrUrArUrArArUrArCrGrCrGrUAT \\
\cline { 2 - 4 } & Antisense & rArUrArCrGrCrGrUrArUrUrArUrArCrGrCrGrArUrUrArArCrGrArC \\
\hline
\end{tabular}

\subsection{Formulation of cationic NISV (CN)}

CN were prepared by microfluidics as described previously [18] using a NanoAssemblr ${ }^{\mathrm{TM}}$ (Benchtop, Precision NanoSystems Inc., Canada). Three different $\mathrm{CN}$ formulations (A-C) were prepared (Table 2). To prepare the $\mathrm{CN}$, the required lipid components at the desired ratios were dissolved in ethanol at a final lipid concentration of $10 \mathrm{mg} / \mathrm{ml}$. The lipid phase was then injected into the first inlet and the aqueous buffer (sterile RNase-free water) into the second inlet of the microfluidic microchannel using disposable syringes. $\mathrm{CN}$ were formulated at a total flow rate of $12 \mathrm{ml} / \mathrm{min}$ and a volumetric flow rate ratio of 3:1 between the aqueous and lipid phase at $50^{\circ} \mathrm{C}$. The resulting $\mathrm{CN}$ dispersions collected from the outlet stream were immediately diluted with sterile RNase-free water in order to reduce the final ethanol content in the preparation to $6.25 \%(\mathrm{v} / \mathrm{v})$. 
Table 2. Composition of CN formulations prepared by microfluidic mixing.

\begin{tabular}{|c|c|c|}
\hline Formulation & Lipid components & Molar ratio \\
\hline A & MPG:Chol:DDAB & $40: 40: 20$ \\
\hline B & MPG:Chol:DDAB & $30: 50: 20$ \\
\hline C & T85:Chol:DDAB & $40: 40: 20$ \\
\hline
\end{tabular}

\section{3. $\mathrm{CN}$ characterisation}

Particle size, polydispersity index (PDI), and zeta potential (ZP) of the $\mathrm{CN}$ formulations were measured by dynamic light scattering (DLS) using a Zetasizer Nano-ZS (Malvern Instruments, UK). The samples were diluted in distilled water at a 1/20 dilution and the measurements were taken at $25^{\circ} \mathrm{C}$.

\subsection{Stability studies of $\mathrm{CN}$}

$\mathrm{CN}$ colloidal systems were tested for their stability over a two month storage at room temperature $\left(25^{\circ} \mathrm{C}\right)$ in a controlled temperature room. $\mathrm{Z}_{\text {Average}}, \mathrm{PDI}$, and $\mathrm{ZP}$ were measured every week.

\subsection{Cytotoxicity of $\mathrm{CN}$ formulations}

A549 cells were grown and maintained in DMEM medium supplemented with $10 \%(\mathrm{v} / \mathrm{v})$ FBS, $1 \%(\mathrm{v} / \mathrm{v})$ L-glutamine and $1 \%(\mathrm{v} / \mathrm{v})$ penicillin-streptomycin. To evaluate the toxicity of the $\mathrm{CN}$, the cells were seeded into a 96 -well plate at a density of $1 \times 10^{4}$ per well in $100 \mu 1$ medium and incubated for $24 \mathrm{~h}$ at $37{ }^{\circ} \mathrm{C}, 5 \% \mathrm{CO}_{2}$ and $100 \%$ humidity. After $24 \mathrm{~h}$, the cells were treated with different concentrations of $\mathrm{CN}(9.77-1250 \mu \mathrm{g} / \mathrm{mL})$ suspended in sterile RNase-free water. Dimethyl sulphoxide (DMSO, 10\% v/v) was used as a positive kill control and untreated cells as the negative control. The plates were incubated for $24 \mathrm{~h}$ and then $20 \mu \mathrm{l}$ of resazurin $(0.1 \mathrm{mg} / \mathrm{ml})$ was added to each well and incubated for a further $24 \mathrm{~h}(48 \mathrm{~h}$ total 
incubation of cells with the $\mathrm{CN}$ formulations).. The quantity of resorufin produced was measured on a SpectraMax M5 plate reader (Molecular Devices, USA) at Ex560/Em590. Cell viability of the treated cells was expressed as a percentage of the untreated control cells.

\subsection{Preparation of $\mathrm{CN} / \mathrm{siRNA}$ nioplexes}

CN/siRNA complexes (termed nioplexes) were prepared as follows: an appropriate volume of siRNA (from $10 \mu \mathrm{M}$ stock) was mixed with the desired $\mathrm{CN}$ formulation (from a $625 \mu \mathrm{g} / \mathrm{ml}$ stock) with pipetting up and down to ensure optimal mixing. The nioplex samples were incubated at room temperature for $30 \mathrm{~min}$ to allow the formation of transfection complexes. For cellular uptake experiments including flow cytometry and fluorescent microscopy, AllStars AF488-labelled Negative Control siRNA (Qiagen, UK) was used to monitor transfection efficiency. For experiments that involved the GFP knockdown in copGFP-A549 cells, siGFP (IDT, Belgium) was used.

\subsection{In vitro cellular uptake}

To study the cellular uptake of nioplexes, A549 cells were seeded in 12-well plates at a density of $1 \times 10^{5}$ cells per well in $1100 \mu \mathrm{L}$ of DMEM medium supplemented with $10 \%(\mathrm{v} / \mathrm{v})$ FBS, $1 \%(\mathrm{v} / \mathrm{v})$ L-glutamine and 1\% (v/v) MEM NEAA (without antibiotics). The following day, $100 \mu \mathrm{l}$ of each of the AF488-labelled Negative Control siRNA-cationic nioplexes (as described in Section 2.6) were added drop-wise to the cells, with gentle plate swirling to ensure uniform distribution of the nioplexes. Transfected cells were incubated for $48 \mathrm{~h}$. The quantitative cellular uptake was measured using a fluorescence-activated cell sorter (FACS). For this purpose, the media was removed and the cells were trypsinised, centrifuged and resuspended in FACS buffer (10\% v/v FBS in PBS) and analysed on a FACS Canto flow cytometer (BD Biosciences; Oxford, UK). Upon acquisition, the cells were gated using forward scatter versus side scatter (FCS vs SSC) to eliminate dead cells and debris. Cells 
$(10,000)$ were collected for each sample and the data analysed with DB FACS Diva software. siRNA alone, $\mathrm{CN}$ alone, and untreated cells were used as controls. The final siRNA concentration after transfection was $10 \mathrm{nM}$. For qualitative uptake measurements, cells were prepared as described above and the cellular uptake of nioplexes were viewed using a Carl Zeiss Axio-Imager Z1 microscope (Zeiss, Germany) under a 20X water immersion lens with a numeric aperture of 0.80 . Fluorescence was excited using a mercury lamp and emission recorded using a fluorescein isothiocyanate (FITC) filter block (485/515-530nm). Analysis of images was carried out with AxioVision 4.8 software.

\subsection{Silencing efficiency studies}

\subsection{1. copGFP-A549 cells preparation}

For the GFP silencing evaluation, copGFP-A549 cells were seeded in 12 -well plates at $1 \times 10^{5}$ cells/ml in $1100 \mu \mathrm{L}$ DMEM medium supplemented with $10 \%(\mathrm{v} / \mathrm{v})$ FBS, $1 \%(\mathrm{v} / \mathrm{v}) \mathrm{L}-$ glutamine, $1 \%$ (v/v) MEM NEAA (without antibiotics) $24 \mathrm{~h}$ before transfection at $37^{\circ} \mathrm{C}, 5 \%$ (v/v) $\mathrm{CO}_{2}$ and $100 \%$ humidity.

\subsubsection{Evaluating GFP silencing by FACS}

To confirm the transfection efficiency of the selected $\mathrm{CN}$ formulations, cells were treated with the desired $\mathrm{CN}$ formulation encapsulating various concentrations of siGFP (10-100 nM final concentration). Control samples contained cells treated with particles alone (mock transfection), siGFP alone, untreated cells, and untreated A549 cells (not producing GFP). The transfection efficiency of the $\mathrm{CN}$ formulations was compared with use of HiPerFect transfection reagent. After transfection, the cells were incubated for $72 \mathrm{~h}$ at $37{ }^{\circ} \mathrm{C}, 5 \% \mathrm{CO}_{2}$ and $100 \%$ humidity, then trypsinised, centrifuged and re-suspended in FACS buffer. A FACS Canto flow cytometer was used to conduct flow cytometry analysis to quantify the \% of GFP expression, the median fluorescence intensity (MFI), and to assess the efficacy of 
GFP silencing by siGFP delivered by the CN formulations. For each sample, 10,000 events were collected. The data obtained were analysed using FACS Diva software. The MFI of GFP was used to calculate the percentage gene silencing using the formula:

$$
\text { Percentage of GFP expression }=\left(\frac{\text { MFI of the transfected cells }}{\text { MFI of the untreated control cells }}\right) \times 100
$$

\subsubsection{Western blotting to determine GFP silencing}

To further assess the downregulation of GFP protein expression in copGFP-A549 cells by siGFP transfected through $\mathrm{CN}$ formulations, cells were treated with various concentrations of siGFP (10-100 nM) transfected using each of the $\mathrm{CN}$ formulations. HiPerFect was used as a positive control. Scrambled negative control siRNA was used at a concentration of $100 \mathrm{nM}$ to prove that transfection of small, non-targeting RNA molecules will not elicit an effect on the cells. Untreated cells, cells treated with naked siGFP, mock transfection (particles alone without siRNA), and cells that did not express GFP were used as controls. The cells were incubated for $72 \mathrm{~h}$, then the media was removed and the cells were lysed with $250 \mu \mathrm{l}$ sample buffer. The GFP protein levels of samples after transfection were determined by Western blotting, normalised with glyceraldehyde 3-phosphate dehydrogenase (GAPDH) levels as a loading control. Twenty five $\mu \mathrm{l}$ of cell lysate was loaded and separated using $10 \%$ MiniPROTEAN $^{\circledR}$ TGX $^{\mathrm{TM}}$ precast gels (Bio-Rad Laboratories Limited, UK), subjected to electrophoresis and then transferred electrophoretically to a nitrocellulose membrane (GE Healthcare Life Sciences, UK). The membrane was incubated with a rabbit polyclonal antibody against copGFP or GAPDH at $4{ }^{\circ} \mathrm{C}$ overnight followed by a secondary antibody anti-rabbit IgG monoclonal antibody and then visualised by a standard enhanced chemiluminescence (ECL) (Thermofisher scientific, UK). Semi-quantification of the bands 
was performed by densitometry using ImageJ public domain software from the National Institutes of Health (http://rsb.info.nih.gov/ij/).

\subsection{Statistical analysis}

All experiments were performed in triplicate and one-way analysis of variance (ANOVA) was used to assess statistical significance. Tukey's multiple comparison test and t-test was performed for paired comparisons. The statistical analysis was performed using Minitab software version 17 . A value of $\mathrm{p}<0.05$ was considered to be statistically significant.

\section{Results}

\subsection{Physiochemical characterisation of $\mathrm{CN}$}

CN prepared by microfluidics were assessed for their size, PDI, and ZP (Table 3). All the prepared formulations were small in size $<60 \mathrm{~nm}$ and had low particle size distribution as indicated by their PDI values $(<0.2)$. As a result of using the cationic lipid DDAB, all the prepared formulations carried an overall positive ZP value.

Table 3. Comparison of particle characteristics of different $\mathrm{CN}$ formulations prepared by microfluidic mixing in terms of size, PDI and ZP. $n=3 \pm S D$

\begin{tabular}{|c|c|c|c|}
\hline Formulation & Size (nm) & PDI & ZP (mV) \\
\hline A & $46.30 \pm 0.18$ & $0.10 \pm 0.02$ & $49.72 \pm 2.80$ \\
\hline B & $49.39 \pm 0.56$ & $0.17 \pm 0.04$ & $51.48 \pm 2.99$ \\
\hline C & $59.16 \pm 1.88$ & $0.19 \pm 0.09$ & $47.45 \pm 2.43$ \\
\hline
\end{tabular}




\subsection{The effects of the lipid composition on overall stability of $\mathrm{CN}$}

CN colloidal systems stability was monitored over two months at room temperature in terms of changes to $\mathrm{Z}_{\mathrm{Average}}$ over time. All the $\mathrm{CN}$ formulations, A-C, were stable with no significant ( $p>0.05)$ change in their particles size (Figure 1).

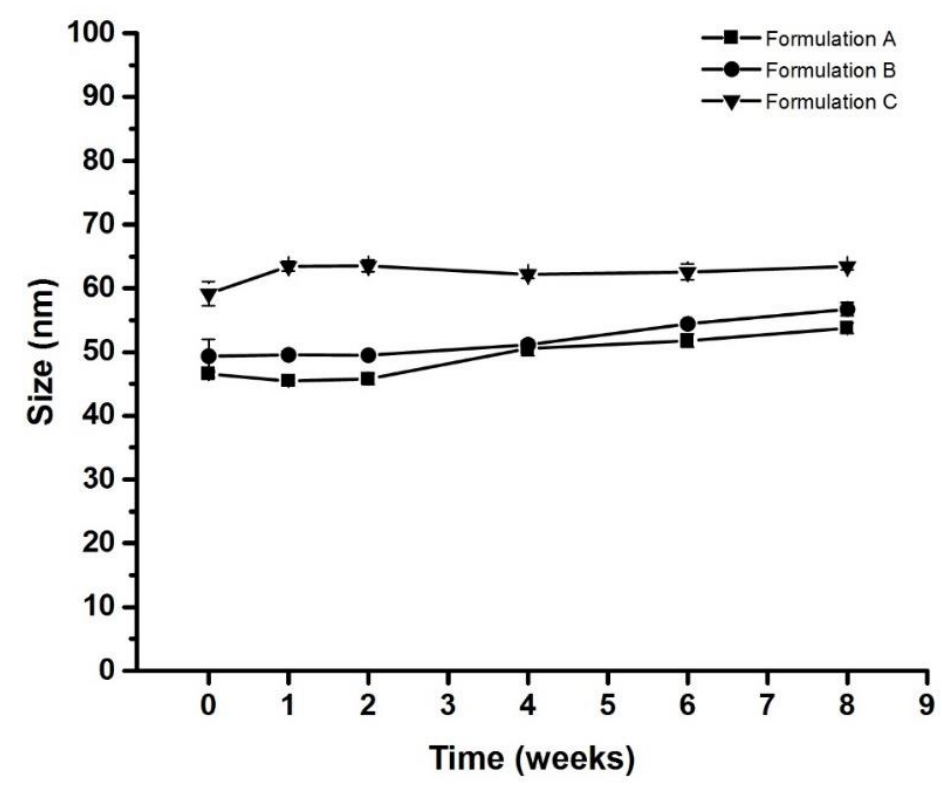

Figure 1. Stability of CN formulations A-C over two months at $25{ }^{\circ} \mathbf{C}$. The data represents the mean $\pm \operatorname{SD}(n=3)$.

\subsection{Cytotoxicity of $\mathrm{CN}$ on the $\mathrm{A549}$ cell line}

Toxicity of the $\mathrm{CN}$ formulations was assessed on A549 cells to quantify cell viability (Figure 2). The cytotoxicity increased as a function of $\mathrm{CN}$ concentration. For all formulations, concentrations equal or below $78.13 \mu \mathrm{g} / \mathrm{ml}$ were well tolerated by cells, with $100 \%$ cells viability. $\mathrm{CN}$ concentration above $312.5 \mu \mathrm{g} / \mathrm{ml}$ induce significant $(\mathrm{p}<0.05)$ cytotoxicity. As a result, all the transfection experiments were carried out at $\mathrm{CN}$ concentrations less or equal to $78.13 \mu \mathrm{g} / \mathrm{ml}$ as a final concentration after transfection to avoid confounding vehicle related cytotoxicity with the gene silencing activity of siRNA. 

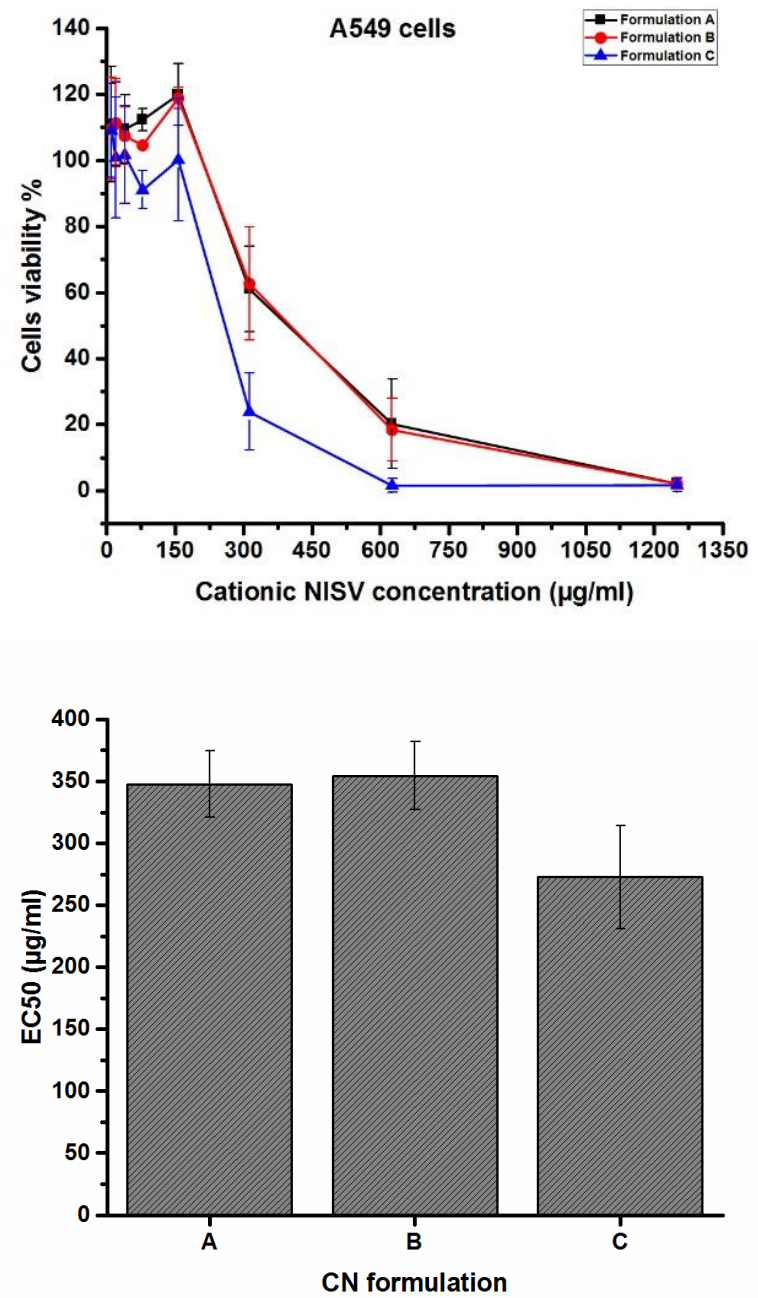

Figure 2. Cytotoxicity of the CN (formulations A-C) on A549 cells and the

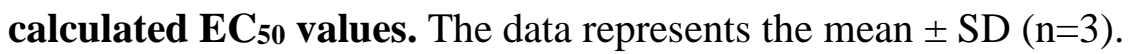

\subsection{Uptake of siRNA nioplexes by A549 cells}

To study the cellular uptake of siRNA encapsulated in CN formulations, A549 cells were treated with the various nioplexes prepared with AF488-labelled Negative Control siRNA. The treated cells were analysed by fluorescence microscopy for qualitative analysis and with FACS for quantitative cellular uptake. 
As can be seen in Figure 3(A), A549 cells did not present any intracellular siRNA signals after incubation with naked siRNA as evidenced by the lack of any green fluorescence associated with the cells. Cells treated with AF488-labelled Negative Control siRNA encapsulated in the three formulations of the $\mathrm{CN}$ A-C showed a strong green fluorescence signal indicating siRNA uptake by the cells. The positive control transfection reagent, HiPerFect, also displayed an uptake of the AF488-labelled Negative Control siRNA. The CN formulations alone, without AF488-labelled Negative control siRNA, showed no fluorescence (images not shown). These results were confirmed by FACS studies, where the fluorescence histogram of the cells incubated with AF488-labelled Negative Control siRNA alone was similar to that of the untreated cells. The histograms for the cells treated with AF488-labelled Negative Control siRNA encapsulated in formulations A-C and with the HiPerFect transfection reagent show a shift in the FITC values compared to the untreated cells (Figure 3(B)) confirming cellular uptake. However, the histogram images indicate a variation in the degree of the curve shift of the three formulations. To further analyse this variation, the percentage cellular uptake for each formulation was measured (Figure 4) and found to be for formulations A and B $93.18 \pm 2.10 \%$ and $93.15 \pm 0.74 \%$, respectively with no significant $(p>0.05)$ difference between them. These percentages were significantly $(\mathrm{p}<0.05)$ higher than the uptake achieved using HiPerFect $(80.08 \pm 1.42 \%)$. The percentage cellular uptake achieved with formulation $\mathrm{C}$ was $73.71 \pm 0.14 \%$, which was significantly $(\mathrm{p}<$ 0.05) lower than formulations A, B, and HiPerFect. However, the uptake achieved with all three formulations and HiPerFect were significantly $(\mathrm{p}<0.05)$ higher than the siRNA uptake when the cells were treated with siRNA alone without any transfection agent. 
A
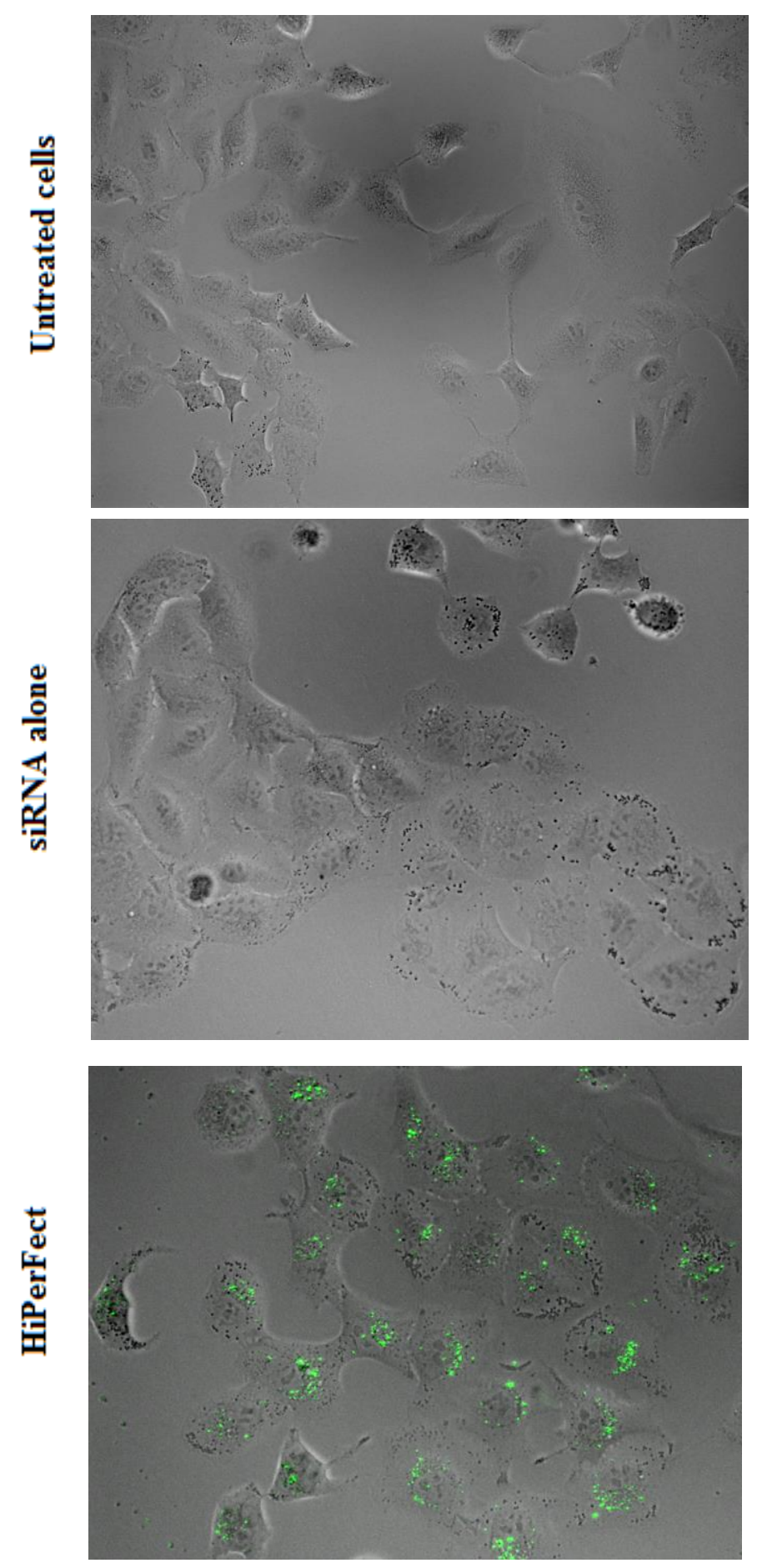

B
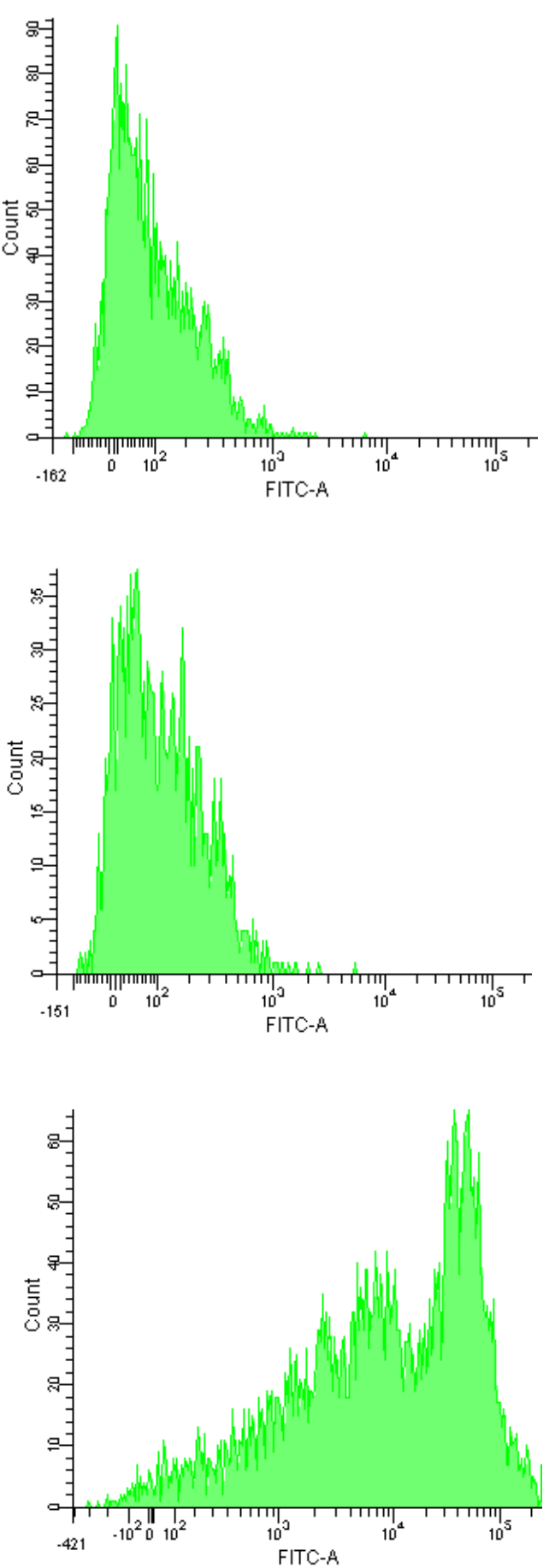

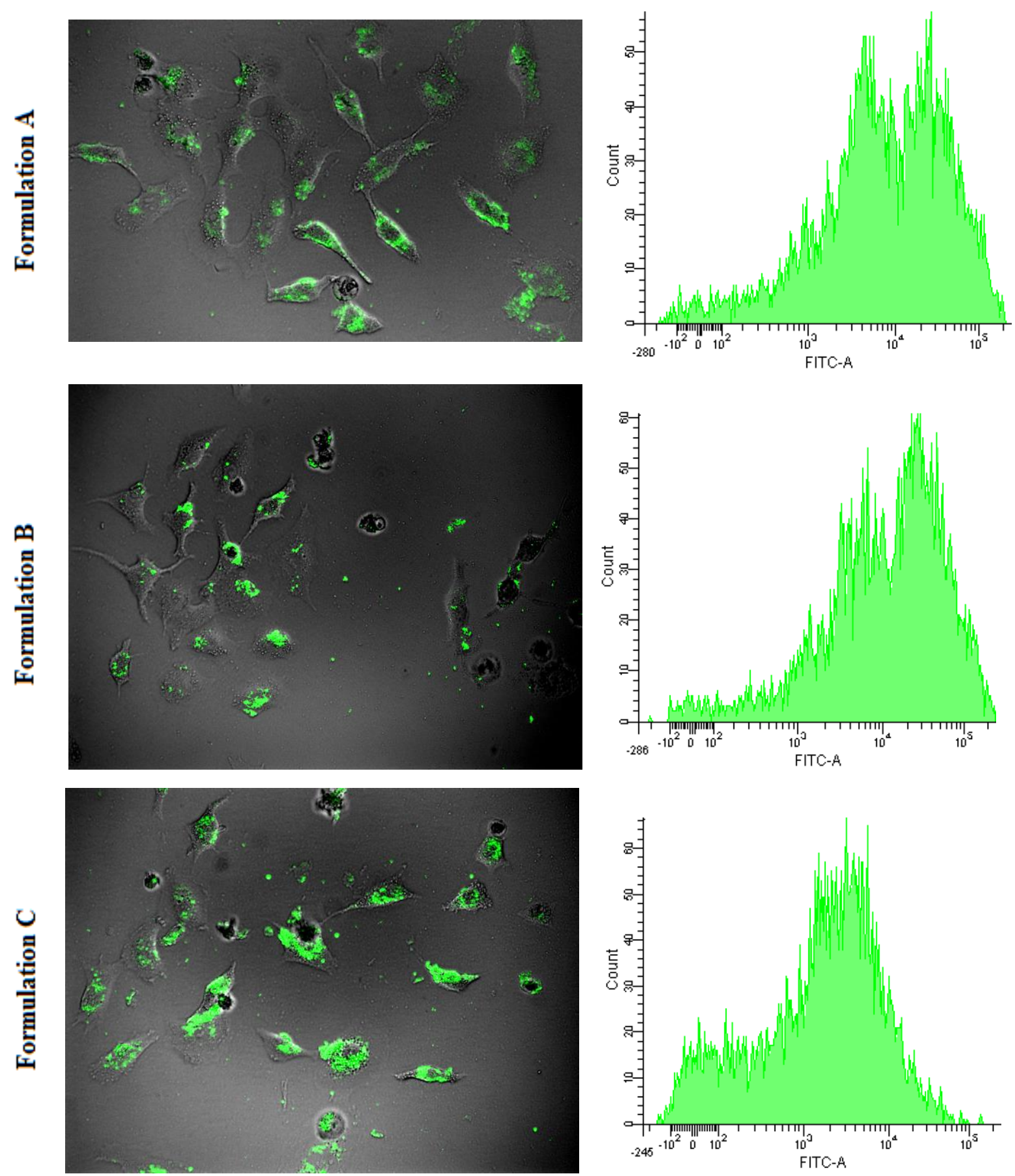

Figure 3 (A) Fluorescent microscopic images (objective lens 20X) and (B) flow cytometry histograms of A549 cell uptake when treated with nioplexes made with AF488-labelled siRNA. Images are representative of three independent images from each sample. The data present means \pm standard deviation $(n=3)$. 


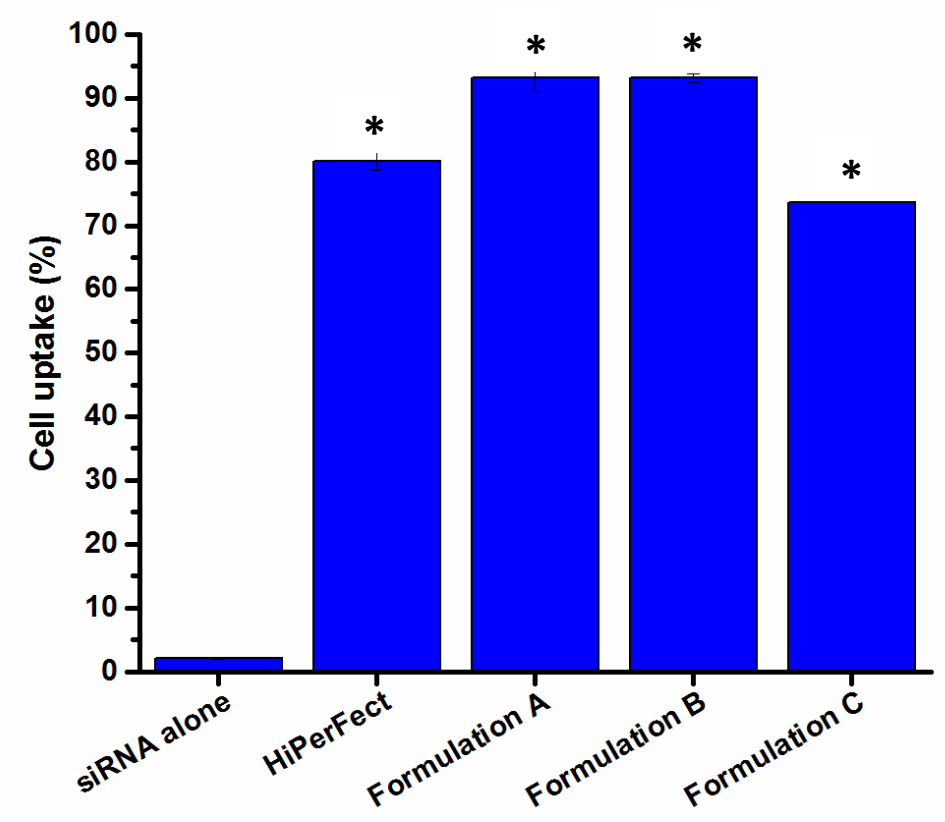

Figure 4. FACS results for the percentage cellular uptake of siRNA by A549 cells when treated with nioplexes encapsulating AF488-labelled siRNA. *Significant $(\mathbf{p}<0.05)$ difference from cells treated with siRNA alone. The data represents means $\pm \mathrm{SD}(\mathrm{n}=3)$.

\subsection{Silencing efficiency studies}

\subsubsection{GFP silencing by FACS}

To assess the GFP knockdown by siGFP delivered by the CN formulations, copGFP-A549 cells were transfected with various concentrations (10-100 nM) of siGFP using formulations A-C to investigate the effect of siRNA dose on transfection activity. siGFP concentrations greater than $100 \mathrm{nM}$ were not evaluated in order to avoid possible off-target effects. The percentage of GFP expression was calculated compared against untreated cells as $100 \%$. As can be seen in Figure 5, all formulations were able to bring down the GFP expression with different percentages. Formulation A was the least effective in silencing GFP with a 
minimum value of GFP expression of $65.79 \pm 9.16 \%$, achieved using $50 \mathrm{nM}$ siGFP. For formulation B, GFP silencing activity increased progressively with siGFP concentration. At 10 and $25 \mathrm{nM}$, the percentages of GFP expression were $78.53 \pm 11.22$ and $82.82 \pm 13.12 \%$ respectively, which was not significantly $(\mathrm{p}>0.05)$ different from formulation $\mathrm{A}$ at the same concentration. However, when the siGFP concentration increased to 50 and $100 \mathrm{nM}$, the GFP expression was brought down to $40.19 \pm 11.68$ and $49.97 \pm 15.05 \%$, respectively, which was significantly $(\mathrm{p}<0.05)$ higher than formulation $\mathrm{A}$ at the same concentrations. A significant down-regulation of GFP expression was determined using formulation C in which the GFP expression was brought to about $30 \%$ compared to untreated cells at all siGFP concentrations. This knockdown efficiency using formulation $\mathrm{C}$ was significantly $(\mathrm{p}<0.05)$ higher than the knockdown achieved using formulations $\mathrm{A}$ and B. For example, at $100 \mathrm{nM}$ siGFP concentrations, the percentage of GFP expression achieved by formulations A-C were 74.58 $\pm 1.53,49.97 \pm 15.04$, and $27.78 \pm 16.51 \%$, respectively, indicating a 3 and 2 -fold higher siRNA transfection efficiency for formulation $\mathrm{C}$ compared with formulations $\mathrm{A}$ and $\mathrm{B}$, respectively. These results for formulation $\mathrm{C}$ were almost the same as that for HiPerFect, where the percentage of GFP expression was around 35\% at all siGFP concentrations used. In contrast, naked siGFP and mock transfection using all formulations showed minimal GFP down-regulation. 

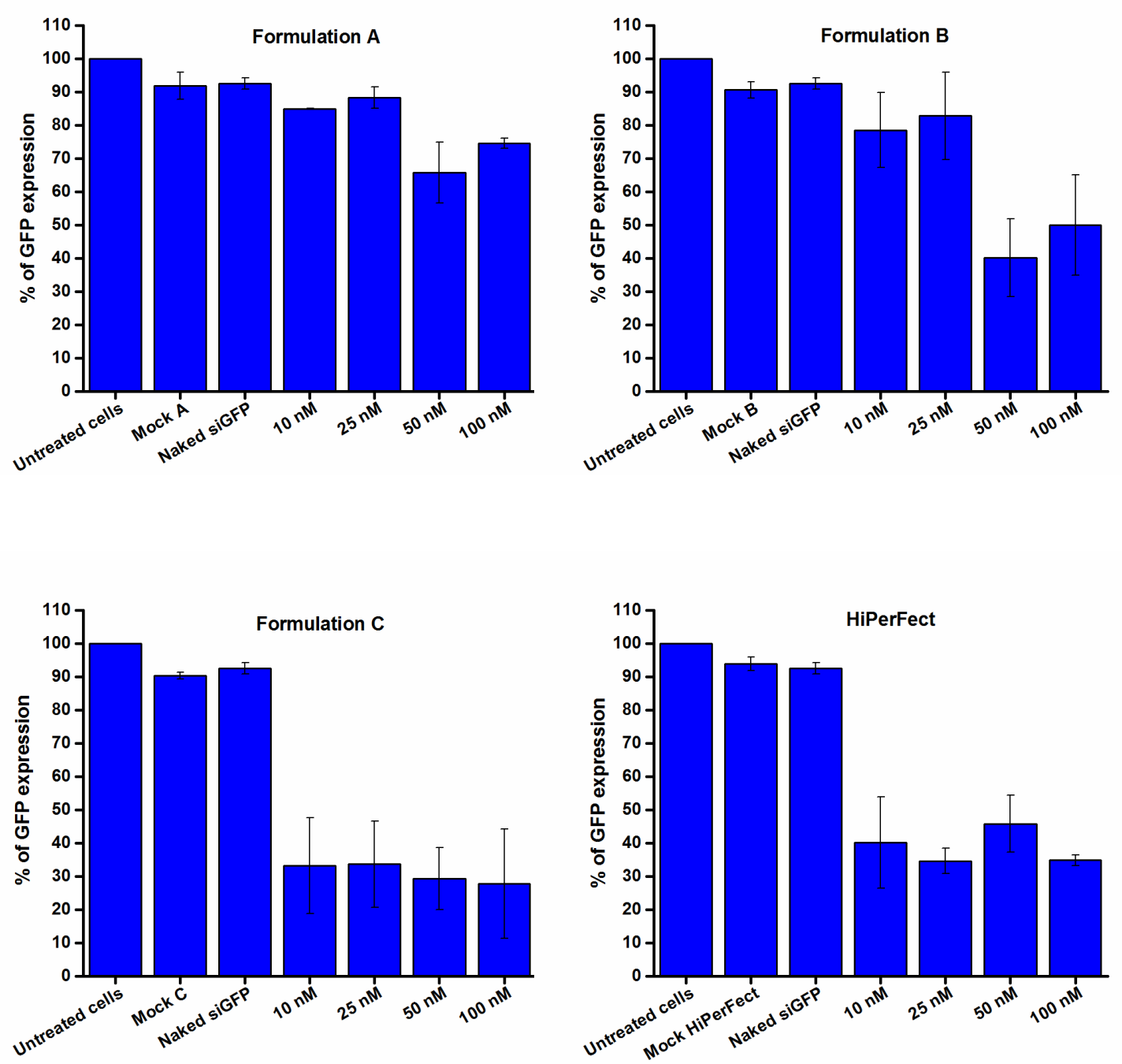

Figure 5. Gene down-regulation analysis in copGFP-A549 cells after transfection with different anti-GFP siRNA (siGFP) concentrations (10-100 nM) transfected with formulations A-C and HiPerFect. GFP expression was quantified by flow cytometry analysis. Values represent the mean $\pm \mathrm{SD}(\mathrm{n}=3)$.

\subsubsection{GFP silencing measured by Western blotting}

To further confirm the results obtained above, the relative GFP levels of treated cells with different siGFP concentrations $(10-100 \mathrm{nM})$ transfected with the three formulations (A-C) 
was evaluated in vitro in copGFP-A549 cells by Western blotting. Similar to FACS results, all three formulations were able to transfect the copGFP-A549 cells and down-regulate the GFP expression by siRNA. As can be seen in Figure 6 (A), formulation C was able to induce the highest GFP knockdown at all the concentrations used compared with formulations A and B. Moreover, mock transfection, naked siGFP, and scrambled negative control siRNA transfected via all formulations did not induce any GFP suppression, which indicates the effectiveness of the $\mathrm{CN}$ formulations and the specificity of the siGFP used. Semiquantification of the bands showed that the GFP expression was $18.79 \pm 5.54 \%$ when the cells were transfected with $10 \mathrm{nM}$ siGFP loaded in formulation $\mathrm{C}$, while cells transfected with higher siGFP concentrations $(25-100 \mathrm{nM})$ with formulation $\mathrm{C}$ had the same GFP expression $(\sim 10 \%)$. These results, achieved by formulation $\mathrm{C}$, were comparable with the GFP inhibition achieved using HiPerFect where the GFP expression was about $10 \%$ at all concentrations used (10-100 nM) (Figure 6 (B)). The percentage GFP expression in cells treated with $10 \mathrm{nM}$ siGFP using formulation B was $64.83 \pm 5.08 \%$, while the GFP expression when the cells were transfected with higher concentrations $(25-100 \mathrm{nM})$ was stable $(\sim 55 \%)$. Formulation A results were comparable with those of formulation B where the GFP expression was $68.80 \pm 6.18 \%$ using $10 \mathrm{nM}$ siRNA, while cells transfected with $25 \mathrm{nM}$ resulted in GFP expression of $55.19 \pm 7.58 \%$. However, higher concentrations of siGFP transfected with formulation A did not achieve higher inhibition of GFP expression. 
(A)

(B)

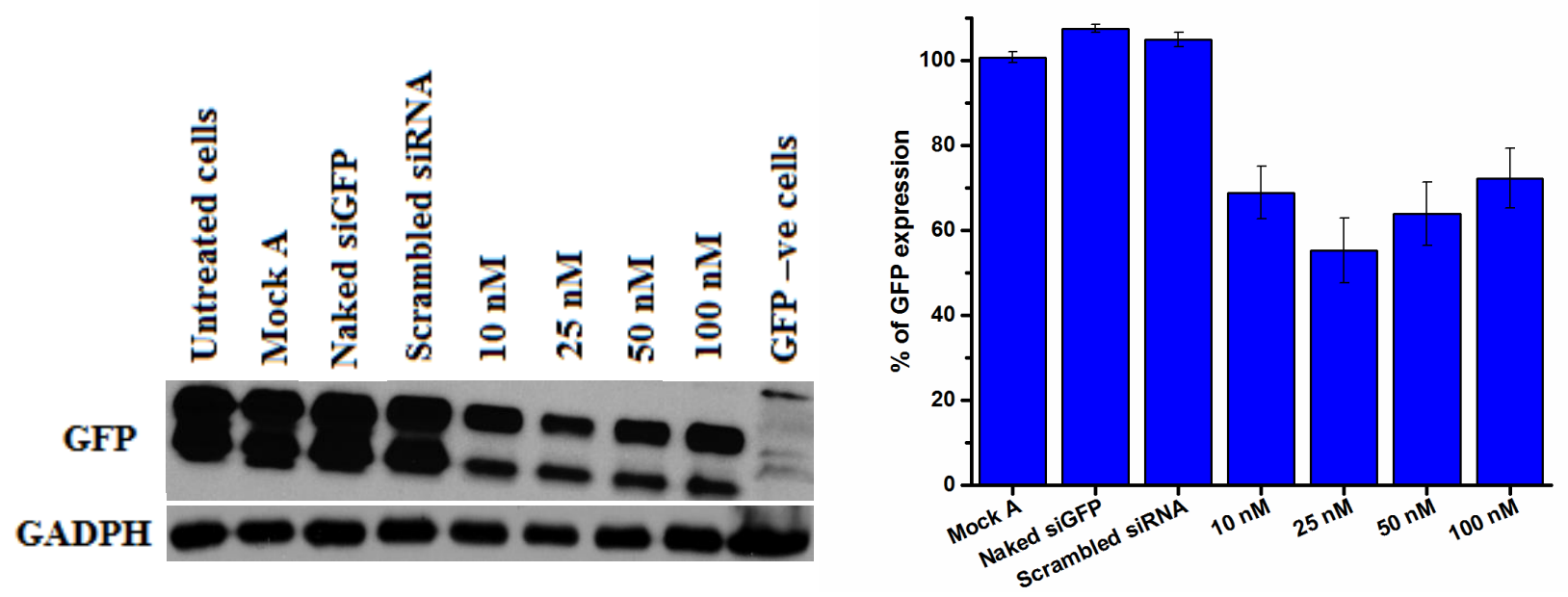

Formulation A

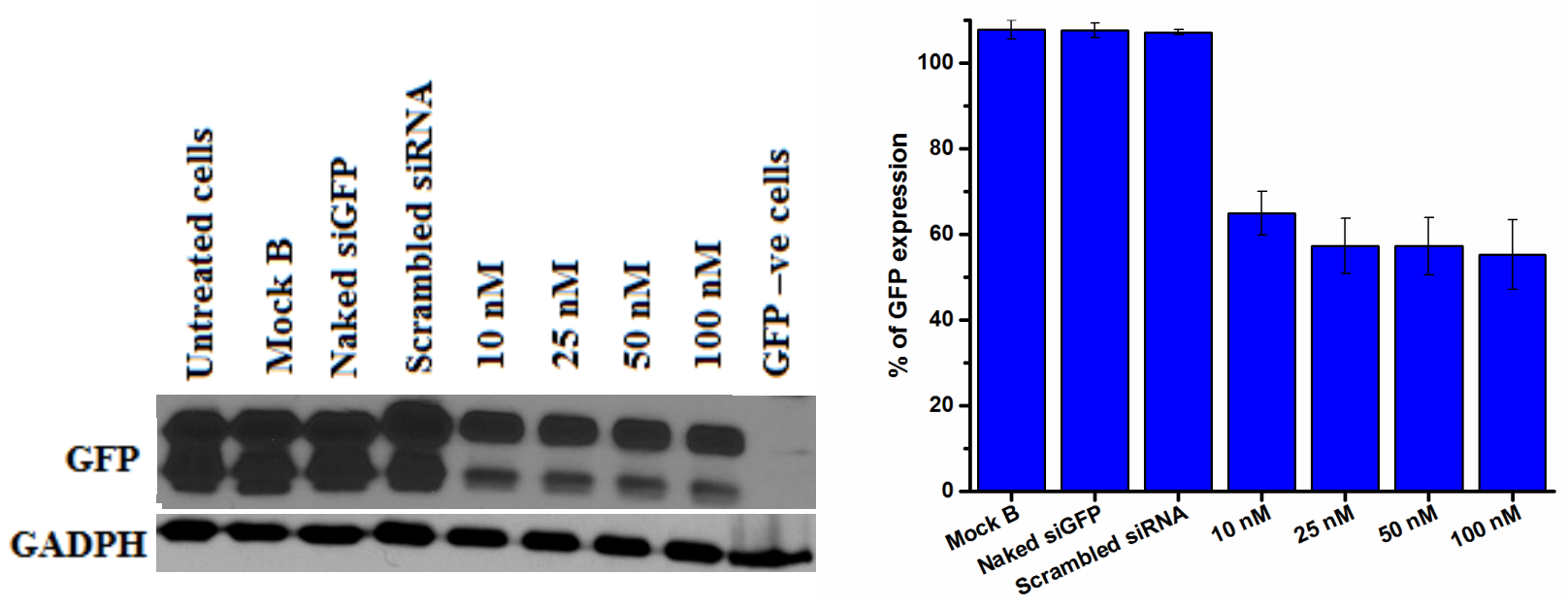

Formulation B 

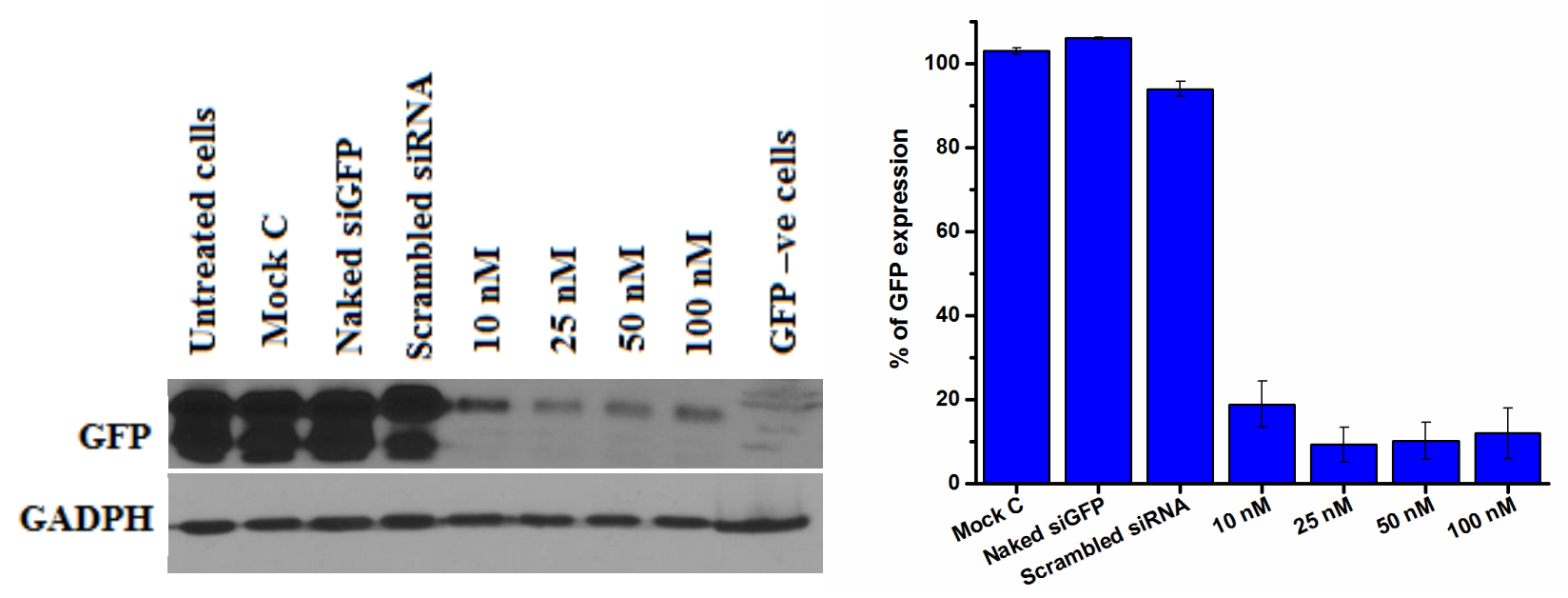

Formulation C
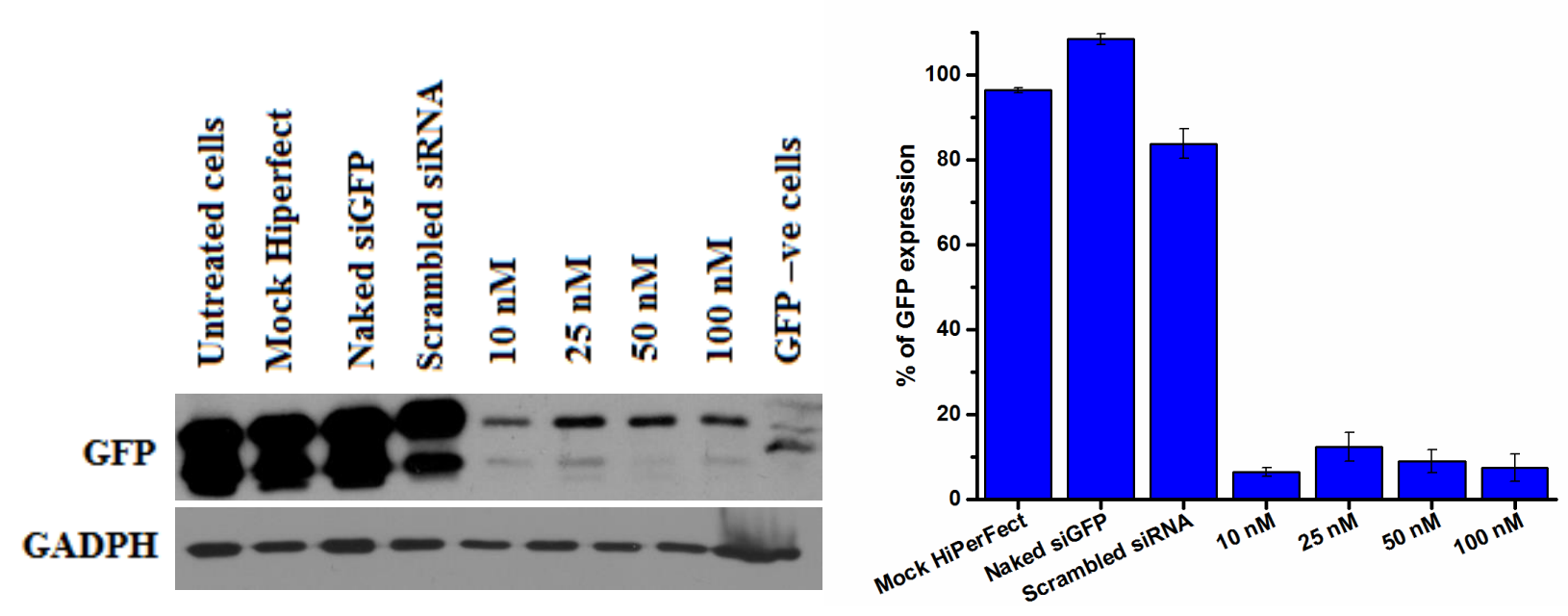

Hiperfect

Figure 6. (A) GFP expression determined by Western blotting after transfecting copGFP-A549 cells with various concentrations $(10-100 \mathrm{nM})$ of anti-GFP siRNA (siGFP) using formulations A-C and HiPerFect. Mock (cells treated with particles only without siRNA), naked siGFP (cells treated with $100 \mathrm{nM}$ siGFP alone without transfection formulation), scrambled siRNA (cells treated with negative control siRNA delivered by the desired formulation at $100 \mathrm{nM}$ concentration), and GFP -ve cells (A549 cells that are not producing GFP). (B) Densitometric analysis of the Western blot shown in (A) determined by ImageJ Software. Results represent the mean \pm SD of three experiments. 


\section{Discussion}

siRNA has significant potential to act as a gene-specific therapeutic agent for the treatment of a wide range of diseases [24]. However, the clinical application of siRNA is limited by the development of effective delivery systems. NISV are gaining more interest as a drug delivery system for various therapeutic agents [18]. Previous work using formulations that combine non-ionic surfactants with phospholipids were used for the delivery of oligonucleotides [25], and for siRNA [24]; however, these formulations were a combination between liposomes and NISV.

The present work explored the potential of NISV as a delivery system for siRNA. Three different formulations were prepared by microfluidics and they were found to be monodisperse and small $(<60 \mathrm{~nm})$ which is a desirable characteristic for drug delivery systems to enhance the accumulation at the tumour site through the enhanced permeability and retention effect [26]. The prepared formulations A-C were shown to have good colloidal stability over two months at $25^{\circ} \mathrm{C}$.

The cytotoxicity of the prepared formulations on the A549 cell line was measured to make sure any gene knock-down was caused by the siRNA used, rather than from any toxic effect of the delivery system. At concentrations less than $78.13 \mu \mathrm{g} / \mathrm{ml}$, all the formulations were non-toxic to the cells and this was consistent with what was reported previously for NISV using other formulations [18]. All the following experiments that include siRNA transfection were carried out at a final concentration at or below this concentration.

The transfection efficiency of the formulations was evaluated as a measure of cellular uptake of siRNA. Cellular uptake analysis is a primary assay that helps to understand part of the transfection process and show the efficiency of the formulations to be internalised when they are in contact with the target cells. Gene knockdown by siRNA is an indication that the 
siRNA has been properly delivered by the vector. However, the transfection process sometimes involves a low number of cells which might be inadequate for gene therapy. Therefore, one of the main objectives of this work was to achieve high percentages of target cell transfection with minimal toxic effects.

To test the effectiveness of formulations A-C in delivering siRNA into the cells and to quantify the cellular uptake, AF488-labelled siRNA was encapsulated into each of the three formulations and transfected into A549 cells. Fluorescent microscopy and flow cytometry were used to assess the cellular uptake. All three formulations were able to deliver the siRNA to the cells as indicated by the strong green fluorescent areas on the microscopy images and by the shift in the fluorescence histograms in the FACS results, compared to the untreated cells. Cellular uptake is influenced by different factors such as particle size, shape, surface charge, and chemistry of the nanoparticles [27]. Regarding the effect of particle size on nanoparticle cellular uptake, several studies reported that a $40-50 \mathrm{~nm}$ diameter is optimal to maximise the cellular uptake in certain mammalian cells $[28,29]$. A lower degree of cellular uptake was noticed for particle sizes above and below this range [30]. In addition, cationic nanoparticles are usually taken up by the cells at a higher rate than anionic particles as a result of interaction of the positive charge with negatively charged domains on the cell membrane [31]. By analysing the characteristics of the $\mathrm{CN}$ formulations $\mathrm{A}-\mathrm{C}$, all three were carried positive charge and were within the desired size range. These favourable characteristics resulted in high cellular uptake for all three formulations, as can be seen in FACS and fluorescent microscope results. The percentage cellular uptake was similar for formulations A and B and lower for formulation C. Formulation A and B are similar in their compositions as both formulations contain the same lipid components in different ratios, while formulation $\mathrm{C}$ is composed of Tween 85 as a non-ionic surfactant instead of MPG used in formulations $\mathrm{A}$ and $\mathrm{B}$. Taking into consideration that all three formulations carry almost 
the same positive charge, this difference in the cellular uptake between the three formulations is attributed to their chemical composition. Another explanation for this difference may be due to the fact that formulation $\mathrm{C}$ is slightly larger in size $(\sim 60 \mathrm{~nm})$ than formulations $\mathrm{A}$ and B (both formulations are $\sim 50 \mathrm{~nm}$ ). This takes formulation $\mathrm{C}$ out of the $40-50 \mathrm{~nm}$ range, which is optimal for cellular uptake.

Following cellular uptake, an effective delivery system must promote endosomal release of siRNA into the cytoplasm for gene silencing [32]. To further examine the efficacy of our formulations in delivering siRNA into cells for the purpose of gene silencing, a series of gene silencing studies on GFP-producing A549 cells (copGFP-A549) to target GFP expression by siGFP were carried out. GFP is a protein that exhibits a bright florescence when exposed to light in the blue to ultraviolet range and is used as a marker of gene expression and protein targeting in intact cells and organisms [33]. CopGFP-A549 is a commercially available A549 cell line that is modified by the supplier to ensure they endogenously express copGFP. A decrease in GFP fluorescence in these cells after transfection indicates endosome release of the siGFP into their cytoplasm where the RNAi knockdown mechanism occurs. By monitoring any changes in fluorescence intensity of the GFP-producing cells after transfection with anti-GFP siRNA, the efficacy of the delivery system can be judged.

Therefore, we analysed the copGFP-A549 cells by FACS after transfection with various concentrations (10-100 $\mathrm{nM}$ ) of siGFP using formulations A-C, with HiPerFect as a positive control. By comparing the fluorescence of the transfected cells with untreated cells, an estimation of the transfection efficiency of the three formulations could be made. FACS analysis showed that all three formulations were able to downregulate GFP expression to varying degrees. Formulation $\mathrm{C}$ was able to suppress GFP expression using siGFP by more than $70 \%$ compared to the untreated cells at all the siRNA concentrations, which was comparable with the GFP knockdown achieved using HiPerFect. The GFP knockdown using 
formulation $\mathrm{C}$ was superior to the GFP knockdown achieved by formulations A and B. These GFP knockdown results using the $\mathrm{CN}$ formulations were higher than the GFP knockdown achieved with other delivery systems. For example, Zhu et al. designed multifunctional polymeric micelles for siRNA delivery and targeting GFP production in copGFP-A549 cells. With their system, the maximum GFP knockdown they achieved was about 55\% compared to untreated cells after one transfection as shown by FACS [34]. Zhou et al. were able to achieve a maximum of $65 \%$ of GFP silencing in human breast cancer cells (MDA-MB-231) using $100 \mathrm{nM}$ siGFP transfected through their SPANosome vesicles [24].

To further understand the transfection efficiency of the $\mathrm{CN}$ formulations, we analysed the transfected cells using Western blotting for more quantitative measurements of the GFP expression. Western blot GFP expression results were consistent with the results obtained by FACS. All the prepared formulations were able to suppress the GFP expression with formulation $\mathrm{C}$ being superior to formulations $\mathrm{A}$ and $\mathrm{B}$. These results demonstrated that the reduction of GFP expression by the CN/siRNA complexes, demonstrated by protein quantification by Western blot, support an RNAi-mediated mechanism of gene silencing after endosome release of siGFP into the cytoplasm where the RNAi mechanism occurs.

Together with the cytotoxicity data, these results also suggests that downregulated gene expression due to non-specific toxic effects of the formulations used can be excluded, since no effect on GFP expression was obvious when the formulations alone (mock) were used as controls which confirms that the observed GFP suppression was due to siGFP transfection instead of vehicle-related cytotoxicity.

It has been reported in the literature that the transfection efficiency by lipid-based nanoparticles strongly depends on the chemical composition of the lipids used $[35,36]$. The data above confirms an efficient delivery of bioactive siRNA into the cytosol after cellular 
uptake and successful release of nanoparticles or siRNA from endosomes or lysosomes can be concluded as a result of the GFP knockdown. Since it was notable that formulation C induced higher gene downregulation than formulations $\mathrm{A}$ and $\mathrm{B}$ using the same siRNA concentrations, the variations in the transfection efficiency between the formulations could be explained, in part, by the different endosomal escape ability of the lipids used [37]. Although formulation $\mathrm{C}$ showed the least cellular uptake compared to the other formulations, this superiority in GFP knockdown compared to formulations A and B suggests that the presence of Tween 85 enhances the endosome escape for formulation $\mathrm{C}$ at a higher rate than the MPG in formulations $\mathrm{A}$ and $\mathrm{B}$.

From all the non-ionic surfactant types, Tween surfactants are one of the most commonly used in the pharmaceutical industry. It includes Tween 20, Tween 60, Tween 80, and Tween 85 depending on the hydrophobic tail present. An attractive property in the structure of all the Tween surfactants is the presence of the hydrophilic polyoxyethylene chain which has been shown to possess a functional interaction with nucleic acids and is used as a gene transfer helper [38]. Endosome escape is one of the major barriers for efficient gene delivery. Tween surfactants are believed to have a fusogenic property similar to dioleoylphosphatidylethanolamine (DOPE), which is one of the most commonly used helper lipids in liposome formulations to facilitate endosome escape of liposomes and its nucleic acids cargo into the cytosol $[39,40]$. From all the Tween surfactant types, Tween 85 is shown to have the highest efficacy when used with NISV for DNA delivery in which the particles formed were proven to have the highest endosome escape compared to NISV prepared with other Tween surfactants [38]. For all these reasons, the presence of T85 in formulation C was believed to enhance endosome release of the siGFP into the cytosol, where the RNAi mechanism occurs, at a higher rate than formulations $\mathrm{A}$ and $\mathrm{B}$, which contributes to the enhanced silencing efficiency of the GFP expression. 
To our knowledge, this is the first report on the use of NISV prepared by microfluidics for the delivery of siRNA. The surfactant vesicles therefore could prove a superior technology platform for therapeutic siRNA delivery. The present work demonstrates that our $\mathrm{CN}$ can be used to effectively deliver siRNA in vitro. Future work including cellular trafficking and in vivo activity evaluation are currently under investigation.

\section{Conclusions}

Here, for the first time the development of $\mathrm{CN}$ prepared by microfluidics for a siRNA delivery was reported. The formulations prepared possessed favourable physical characteristics and mediated efficient cytosolic delivery of siRNA. From the formulations tested, formulation $\mathrm{C}$ that was composed of Tween 85 as a non-ionic surfactant, showed superiority over the other two formulations, composed with MPG as the surfactant. The transfection efficiency of formulation $\mathrm{C}$ was shown to be comparable with the cationic transfection reagent, HiPerFect. In conclusion, these novel vectors constitute promising agents for delivery of siRNA and deserve further investigation.

\section{Acknowledgments}

The authors would like to acknowledge the Jordanian Ministry of Higher Education and Scientific Research and Yarmouk University in Jordan for funding this work. Also, the author would like to thank Mr. Brian Cartlidge from the Department of Biomedical Engineering for help with the fluorescence microscopy. 


\section{References}

1. Fire, A., et al., Potent and specific genetic interference by double-stranded RNA in Caenorhabditis elegans. nature, 1998. 391(6669): p. 806-811.

2. Hammond, S.M., Dicing and slicing. FEBS letters, 2005. 579(26): p. 5822-5829.

3. Ameres, S.L., J. Martinez, and R. Schroeder, Molecular basis for target RNA recognition and cleavage by human RISC. Cell, 2007. 130(1): p. 101-112.

4. $\quad$ Elbashir, S.M., et al., Duplexes of 21-nucleotide RNAs mediate RNA interference in cultured mammalian cells. nature, 2001. 411(6836): p. 494-498.

5. McCaffrey, A.P., et al., Gene expression: RNA interference in adult mice. Nature, 2002. 418(6893): p. 38-39.

6. Miele, E., et al., Nanoparticle-based delivery of small interfering RNA: challenges for cancer therapy. Int J Nanomedicine, 2012. 7: p. 3637-3657.

7. Lee, S., et al., The role of c-FLIP in cisplatin resistance of human bladder cancer cells. The Journal of urology, 2013. 189(6): p. 2327-2334.

8. Goldberg, M.S., siRNA Delivery for the treatment of ovarian cancer. Methods, 2013. 63(2): $p$. 95-100.

9. Taratula, O., et al., Nanostructured lipid carriers as multifunctional nanomedicine platform for pulmonary co-delivery of anticancer drugs and siRNA. Journal of Controlled Release, 2013. 171(3): p. 349-357.

10. Zheng, Y., et al., PTK6 activation at the membrane regulates epithelial-mesenchymal transition in prostate cancer. Cancer research, 2013. 73(17): p. 5426-5437.

11. Resnier, P., et al., A review of the current status of siRNA nanomedicines in the treatment of cancer. Biomaterials, 2013. 34(27): p. 6429-6443.

12. Higuchi, Y., S. Kawakami, and M. Hashida, Strategies for in vivo delivery of siRNAs. BioDrugs, 2010. 24(3): p. 195-205.

13. Kim, W.J. and S.W. Kim, Efficient siRNA delivery with non-viral polymeric vehicles. Pharmaceutical research, 2009. 26(3): p. 657-666.

14. David, S., et al., Non-viral nanosystems for systemic siRNA delivery. Pharmacological research, 2010. 62(2): p. 100-114.

15. Li, S.-d. and L.-y. Huang, Nonviral gene therapy: promises and challenges. Gene therapy, 2000. 7(1): p. 31.

16. Obeid, M.A., et al., Comparison of the physical characteristics of monodisperse non-ionic surfactant vesicles (NISV) prepared using different manufacturing methods. International journal of pharmaceutics, 2017. 521(1): p. 54-60.

17. Marianecci, C., et al., Niosomes from 80s to present: the state of the art. Advances in colloid and interface science, 2014. 205: p. 187-206.

18. Obeid, M.A., et al., The effects of hydration media on the characteristics of non-ionic surfactant vesicles (NISV) prepared by microfluidics. International Journal of Pharmaceutics, 2016.

19. Manosroi, A., et al., Stability of luciferase plasmid entrapped in cationic bilayer vesicles. International journal of pharmaceutics, 2008. 356(1): p. 291-299.

20. Perrie, Y., et al., Surfactant vesicle-mediated delivery of DNA vaccines via the subcutaneous route. International journal of pharmaceutics, 2004. 284(1): p. 31-41.

21. Paecharoenchai, O., et al., Nonionic surfactant vesicles for delivery of RNAi therapeutics. Nanomedicine, 2013. 8(11): p. 1865-1873.

22. Sun, M., et al., Enhanced efficacy of chemotherapy for breast cancer stem cells by simultaneous suppression of multidrug resistance and antiapoptotic cellular defense. Acta biomaterialia, 2015. 28: p. 171-182. 
23. Zhou, C., et al., Comparative cellular pharmacokinetics and pharmacodynamics of siRNA delivery by SPANosomes and by cationic liposomes. Nanomedicine: Nanotechnology, Biology and Medicine, 2013. 9(4): p. 504-513.

24. Zhou, C., et al., SPANosomes as delivery vehicles for small interfering RNA (siRNA). Molecular pharmaceutics, 2012. 9(2): p. 201-210.

25. Huang, Y.-Z., et al., Cationic liposomes modified with non-ionic surfactants as effective nonviral carrier for gene transfer. Colloids and Surfaces B: Biointerfaces, 2006. 49(2): p. 158-164.

26. Yingchoncharoen, P., D.S. Kalinowski, and D.R. Richardson, Lipid-Based Drug Delivery Systems in Cancer Therapy: What Is Available and What is Yet to Come. Pharmacological Reviews, 2016. 68(3): p. 701-787.

27. Fröhlich, E., The role of surface charge in cellular uptake and cytotoxicity of medical nanoparticles. Int J Nanomedicine, 2012. 7(1): p. 5577-91.

28. Chithrani, B.D. and W.C. Chan, Elucidating the mechanism of cellular uptake and removal of protein-coated gold nanoparticles of different sizes and shapes. Nano letters, 2007. 7(6): p. 1542-1550.

29. Lu, F., et al., Size effect on cell uptake in well-suspended, uniform mesoporous silica nanoparticles. Small, 2009. 5(12): p. 1408-1413.

30. Albanese, A., P.S. Tang, and W.C. Chan, The effect of nanoparticle size, shape, and surface chemistry on biological systems. Annual review of biomedical engineering, 2012. 14: p. 1-16.

31. Ge, Y., et al., Effect of surface charge and agglomerate degree of magnetic iron oxide nanoparticles on KB cellular uptake in vitro. Colloids and Surfaces B: Biointerfaces, 2009. 73(2): p. 294-301.

32. Varkouhi, A.K., et al., Endosomal escape pathways for delivery of biologicals. Journal of Controlled Release, 2011. 151(3): p. 220-228.

33. Tsien, R.Y., The green fluorescent protein. Annual review of biochemistry, 1998. 67(1): $p$. 509-544.

34. Zhu, L., et al., Matrix metalloproteinase 2-sensitive multifunctional polymeric micelles for tumor-specific co-delivery of siRNA and hydrophobic drugs. Biomaterials, 2014. 35(13): p. 4213-4222.

35. Chung, H., et al., Oil components modulate physical characteristics and function of the natural oil emulsions as drug or gene delivery system. Journal of Controlled Release, 2001. 71(3): p. 339-350.

36. Ojeda, E., et al., The role of helper lipids in the intracellular disposition and transfection efficiency of niosome formulations for gene delivery to retinal pigment epithelial cells. International journal of pharmaceutics, 2016. 503(1): p. 115-126.

37. Pozzi, D., et al., Mechanistic evaluation of the transfection barriers involved in lipid-mediated gene delivery: interplay between nanostructure and composition. Biochimica et Biophysica Acta (BBA)-Biomembranes, 2014. 1838(3): p. 957-967.

38. Huang, Y., et al., Polysorbate cationic synthetic vesicle for gene delivery. Journal of Biomedical Materials Research Part A, 2011. 96(3): p. 513-519.

39. Liu, F., et al., New cationic lipid formulations for gene transfer. Pharmaceutical research, 1996. 13(12): p. 1856-1860.

40. Koltover, l., et al., An inverted hexagonal phase of cationic liposome-DNA complexes related to DNA release and delivery. Science, 1998. 281(5373): p. 78-81. 